\title{
CHARACTERISTIC X-RAY EMISSION AS A FUNCTION OF THE APPLIED VOLTAGE.
}

By BERGEN Davis.

$\mathrm{R}^{\mathrm{E}}$ CENT experiments of D. L. Webster ${ }^{1}$ show that the energy of emission of characteristic (line) radiation from a rhodium target increases rapidly as the voltage applied to the Coolidge X-ray tube is increased. The radiation is not produced at all unless the voltage is greater than a minimum $V_{0}$. This minimum voltage is slightly greater than that corresponding by the quantum relation to the frequency of the $\beta$ line ( $K$-radiation). This law is undoubtedly true also for the characteristic X-ray emission from other elements.

It may be of some interest and value to investigate how this emission should depend on the voltage from a consideration of well-established physical facts. The following facts relating to the problem may be regarded as established by experiment.

(a) The characteristic line emission is zero for all voltages less than a critical voltage $V_{0}$.

(b) The voltage $V_{0}$ at which the characteristic radiation is produced is that corresponding by the quantum relation, $V_{0} e=h n$, to a frequency slightly greater than that of the $K_{\beta}$ radiation of the element.

(c) At voltages equal to and greater than $V_{0}$ both the $\alpha$ and $\beta$ lines of the $K$ radiation are emitted. These two lines increase rapidly in intensity with the voltage, their ratios remaining approximately constant.

(d) X-radiation is not only emitted from the surface atoms of the target, but also from the atoms beneath the surface when they are impacted by the electrons of the cathode stream. ${ }^{2}$

(e) The electrons penetrate a short distance into the surface of the target, but their velocity diminishes rapidly with depth of penetration.

( $f$ ) The emitted X-rays are absorbed on their passage through matter. This absorption depends on the thickness and nature of the material traversed.

The only hypotheses adopted will be directly in keeping with the Bohr theory of the atom. The Bohr picture of the atomic mechanism, which is so successful in the case of the ordinary radiation from hydrogen,

${ }^{1}$ Phys. Rev., June, rgi6.

${ }^{2}$ Kaye, X-rays, p. 40. 
is almost equally successful in the case of characteristic $\mathrm{X}$-radiation, since the frequencies of the $K_{a}$ radiation from many elements may be readily calculated by the Bohr equation for the hydrogen atom.

According to this theory the frequency of the radiation emitted by an atom depends on the change of the potential energy of one or more electrons of the atom with respect to a central nuclear charge. This change of potential energy is radial with respect to the central nucleus.

It will be considered that radiation can only be excited by the transfer of energy from the impacting electron along a radius with respect to the center of the atom.

The mechanism of the atomic nucleus will be considered to emit a quantum of energy whenever an impacting electron possesses such velocity that the energy due to the radial component of this velocity shall be equal to or exceed the minimum energy $\left(V_{0} e\right)$ required for the excitation of the particular radiation. The work done along the radius must be equal to or exceed the quantity $\left(V_{0} e\right)$ where $V_{0}$ is the least voltage that will excite the characteristic radiation.

Let $N$ represent the number of electrons striking the surface of the target per second. Let $B$ represent the probability of any one electron making impact with or coming within the spheres of influence of the atomic nuclei in unit distance. The number of such impacts in a distance $d x$ will be

$B N d x$.

The X-radiation appears to have its origin in the nucleus of the atom. It is considered that the impacting electron in order to excite the radiating mechanism of this nucleus must come within an undefined region about the nucleus which will be referred to as the sphere of influence of the nucleus. The probability $B d x$ of an electron striking one of these nuclear spheres in a path $d x$ is quite small.

Not all of these $B N d x$ impacts with the nuclei will excite radiation, but a fraction of them will do so. The capacity to excite radiation depends on the nature of the impact. Only those impacts will be effective in which the energy due to the radial component of the velocity is equal to or exceeds a minimum $\left(V_{0} e\right)$. This assumption which has previously been applied to ionization by impact $t^{1}$ is, as has just been pointed out, in agreement with the Bohr theory of the atom.

The fraction of the $B N$ electrons that are effective may be readily found by consideration of Fig. I.

For the purpose of presenting a picture to the mind one may tentatively regard an atomic nucleus to be represented at $C$, and the bounding sphere

1 Phys. Rev., Jan., I907. An. d. Physik., Band 42, r9ı3. 
of influence within which the electrons must penetrate in order to excite radiation to be represented by abed.

If the velocity of the electrons on approaching this bounding sphere is that corresponding to $V_{0}$, only those that make impact along the line $h$ will be capable of producing the radiation. All other electrons will have a radial component too small to be effective. As* the voltage is increased, electrons approaching the nucleus further from the atomic pole $a$, as along $f g$, may have a radial component at least equal to $V_{0}$. As the voltage is further increased electrons approaching along a line as far from the pole as $m n$ may be effective. The fraction of the

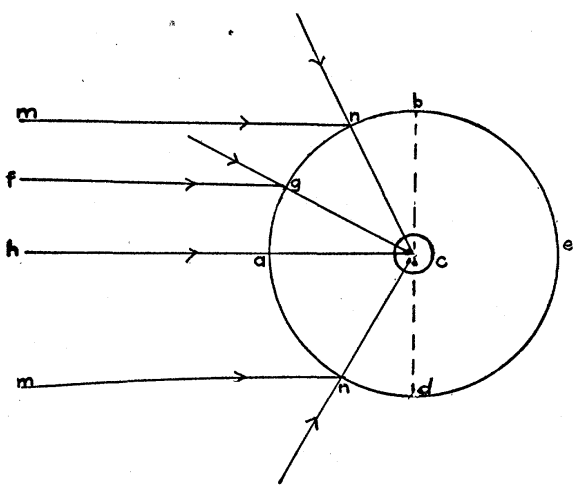

Fig1. impacts that will be effective is the ratio of the cross-section of the zonal area $n n$ to the area of cross-section of the nuclear sphere $b d$. This ratio may be expressed in terms of the voltage $V$ and is

$$
\frac{V-V_{0}}{V}
$$

Of $B N d x$ impacts in a region $d x$ at a depth $x$ within the target the fraction

$$
\frac{V_{x}-V_{0}}{V_{x}}
$$

will be effective in producing radiation where $V_{x}$ is the voltage corresponding to the velocity that an electron may have at a depth $x$ beneath the surface of the target.

Each of these effective impacts will emit a quantum of energy of some frequency. All of the X-radiation does not appear at one frequency, but there are other frequencies emitted in addition to the stronger $\alpha$ and $\beta$ radiations. The electrons in general do not possess sufficient energy to excite both the $\alpha$ and $\beta$ lines, since the energy required would be $\left(h n_{a}+h n_{\beta}\right)$.

Of all the effective impacts, a fraction will produce a disturbance that results in the emission of radiation of one frequency, the $K_{\alpha}$ radiation for example. The fractional part of the total effective impacts that result in the production of radiation of frequency $n_{a}$ will be designated 
by $E_{\alpha}$. This constant $E_{a}$ will be more fully discussed in the last paragraph of this section of the paper. Each of these

$$
E_{\alpha} B N \frac{V_{x}-V_{0}}{V_{x}} d x
$$

impacts will produce a quantum of energy $h n_{a}$. The energy radiated will be

$$
d I_{0}=E_{a}\left(h n_{a}\right) B N \frac{V_{x}-V_{0}}{V_{x}} d x .
$$

The law of decrease of the velocity of electrons penetrating a metallic surface has been derived by Sir J. J. Thomson ${ }^{1}$ and experimentally confirmed by Whiddington. ${ }^{2}$ This law is expressed by the equation

$$
v_{x}^{4}=v^{4}-a x,
$$

where $v_{x}$ is the velocity at a depth $x$ and $a$ is a constant depending on the nature of the material. This equation may be expressed in terms of the corresponding kilo-volts $V$ and becomes

$$
V_{x}^{2}=V^{2}-b x,
$$

where $b$ is the corresponding value of the constant $a$.

Equation (I) may then be written

$$
I_{0}=E_{a}\left(h n_{a}\right) B N\left[\int d x-\int \frac{V_{0} d x}{\left(V^{2}-b x\right)^{1 / 2}}\right] .
$$

The X-rays when emitted from a region $d x$ at a depth $x$ below the surface will be subject to absorption in passing up through the material of the target. The quantity emitted from any depth $x$ will be

$$
I=I_{0} e^{-\mu c x}
$$

where $\mu$ is the coefficient of absorption of the material for the radiation of the given frequency, and $c x$ is the thickness traversed by the rays in emerging from the target. The radiation actually emitted becomes:

$$
I=E_{a}\left(h n_{\alpha}\right) B N\left[\int_{0}^{R} e^{-\mu c x} d x-V_{0} \int_{0}^{R}\left(V^{2}-b x\right)^{-1 / 2} e^{-\mu c x} d x\right] .
$$

The limit $R$ is the range of the electrons before their velocity is reduced to that corresponding to the voltage $V_{0}$. From equation (3)

and

$$
V_{0}^{2}=V^{2}-b R \text {, }
$$

$$
R=\frac{V^{2}-V_{0}^{2}}{b}
$$

${ }^{1}$ Conduction Through Gases, p. 378

2 Proc. Roy. Soc., Vol. 86, I912. 
The indefinite integral of (5) is not obtainable, but a close approximation may be obtained by expanding the exponential term. Since this expanded term is rapidly convergent for the values $\mu c x$ here required it is necessary to retain only a few terms of the series. The resulting integral is

$$
\begin{aligned}
I= & E_{a}\left(h n_{a}\right) \frac{B N}{b}\left[\frac{b}{c \mu}\left(\mathrm{I}-e^{-(c \mu \mid b)\left(V^{2}-V_{0}^{2}\right)}\right)\right. \\
& -2 V_{0}\left(V-V_{0}\right)+\frac{2}{3} \frac{c \mu}{b} V_{0}\left\{2 V^{3}-V_{0}\left(V^{2}+V_{0}^{2}\right)\right\} \\
& -\frac{V_{0}}{\mathrm{I}_{5}}\left(\frac{c \mu}{b}\right)^{2}\left\{8 V^{4}\left(V-V_{0}\right)-4 V^{2} V_{0}\left(V^{2}-V_{0}^{2}\right)\right. \\
& \left.\left.-3 V_{0}\left(V^{2}-V_{0}^{2}\right)^{2}\right\}\right] . \quad(7)
\end{aligned}
$$

The variable part of this equation contains no arbitrary constants. The constants $b$ and $c \mu$ may be obtained by independent experiment.

In order to compare this equation with experiment, I have taken some results just obtained by Mr. B. A. Wooten (not yet published) for the emission of the $K$ characteristic ( $\alpha$ line) radiation from molybdenum. The difficulty of making this comparison arises from the fact that no experimental results are at hand either for the decrease of velocity of electrons (constant $b$ ) or for the absorption of rays of this particular frequency (constant $\mu$ ) for molybdenum.

The constant $a$ has been determined by Whiddington ${ }^{1}$ for gold and aluminium.

$$
\begin{aligned}
& a=7.32 \times 10^{42} . \\
& a=2.54 \times 10^{43} .
\end{aligned}
$$

If the density of the material be represented by $\rho$

$$
\begin{aligned}
& \frac{a}{\rho}=2.83 \times \mathrm{IO}^{42} . \\
& \frac{a}{\rho}=\mathrm{I} .33 \times \mathrm{IO}^{42} .
\end{aligned}
$$

The stopping power of a metal does not appear to be directly related to its density. One can only estimate its value for molybdenum. In the absence of experimental data I shall tentatively assume a value between that found for gold and aluminium.

Taking $a / \rho=2.2 \times 10^{42}$, the value of $a$ is $1.9 \times 10^{43}$. The corresponding value of this constant when the velocity is expressed in terms of kilovolts is

$$
1 \text { Loc. cit. }
$$

$$
b=\mathrm{I} .5 \times 1 \mathrm{IO}^{6} .
$$


The absorption coefficient $\mu$ of molybdenum for its own $K$ characteristic radiation has not been determined, but the coefficient of absorption of these rays in silver is given by $\mathrm{Kaye}^{1}$ as

$$
\frac{\mu}{\rho}=24 \cdot 4
$$

Since molybdenum does not differ much from silver in atomic weight and density, this will be provisionally taken as the absorption coefficient

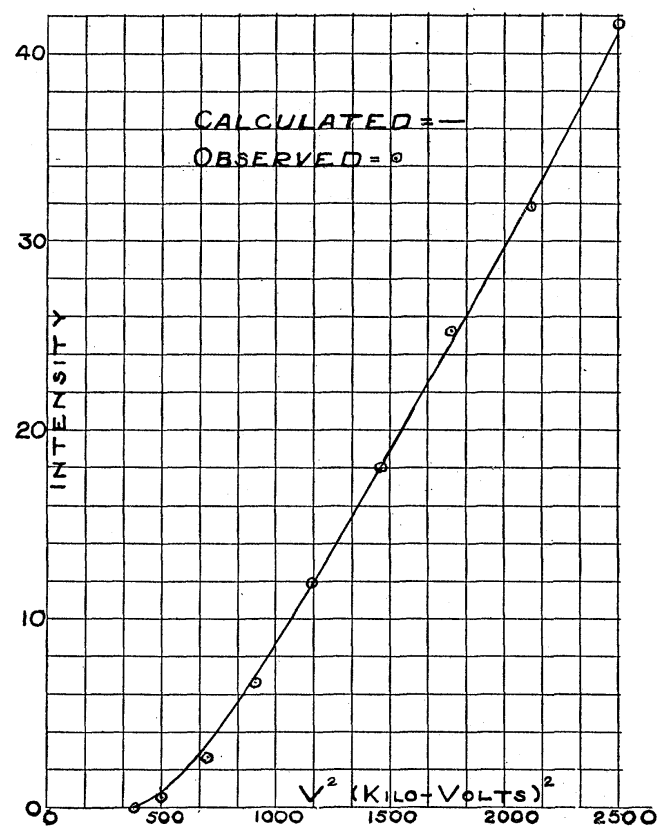

Fig. 2. of molybdenum for its own characteristic radiation.

$$
\mu=200 .
$$

In the particular experiments of Wooten the X-rays were taken from the target at such an angle that the path of the rays emerging from the target was about I.5 times the path $(x)$ of the penetrating electrons that produced them. The constant $c$ is 1.5 . The group constant $b / c \mu$ of equation (7) has the approximate numerical value 5,000 .

The theoretical equation as derived is calculated and plotted in Fig. 2. The curves are plotted with the square of the applied voltage (kilovolts) as abscissæ, since this makes the greater part of the curve nearly a straight line. The circled points represent the observed results obtained by Wooten.

The form of the calculated curve is similar to that obtained by experiment. Since the variable part of the equation contains no arbitrary constants, the assumptions underlying its derivation appear to be justified.

The energy of emission of different elements may be expected to increase rapidly with the atomic number. The energy emitted is proportional to $h n$, where $n$ is a frequency slightly greater than that of the $K \beta$ characteristic of the element, and this frequency is nearly proportional

1 X-rays, Kaye, p. 138. 
to the square of the atomic number. If the constant $E_{a}$ were of the same value for all elements, this increase in emissivity would be approximately proportional to the quantum $h n$.

The factor $B$, which is a constant for any one element, may depend on the atomic number. The introduction of this constant is required, since it is assumed that every effective electron at impact will give rise to a quantum of radiant energy. The fraction of the total electrons of the cathode stream that are effective must be very small. The greater part of them dissipate their energy directly in the production of heat, or indirectly by the excitation of other types of radiation by disturbance of the electrons in the outer regions of the atom. It is possible that the greater part of the transfer of kinetic energy of the impacting electrons to the atoms does not take place directly through the interchange of momentum, but they may excite radiation in the ultra-violet, visible and infra-red regions by disturbance of the more loosely bound electrons of the atom. This radiation is absorbed by the atoms and finally appears as heat.

Equation (7) without the constant $E_{a}\left(h n_{a}\right)$ would represent the number of impacts of the electrons that have a radial component of velocity equal to or greater than that corresponding to the critical voltage $V_{0}$. All the energy of these effective impacts cannot appear as radiation of any one frequency, $n_{c}$ for example (where $n_{c}$ is the frequency corresponding to the critical voltage $\left.V_{0}\right)$. There are at least two strong lines $(\alpha$ and $\beta$ ) and a number of weaker lines in the $K$-radiation, also there are a number of lines in the $L$ characteristic radiation. In addition there is the general radiation of the "continuous" X-ray spectrum. Each effective electron at impact gives rise to only one quantum of energy, so that the same electron cannot excite the characteristic lines as well as the general radiation. There must be some statistical partition between the number that give rise to each type of radiation.

The constant $E_{a}$ expresses the fraction of the effective impacts that excite radiation of one frequency and the combined constant $E_{a}\left(h n_{\alpha}\right)$ expresses the fraction of the radiated energy produced by the effective $B N$ electrons that appear as radiation of one frequency, the $K_{a}$ radiation in the present discussion.

This partition or distribution of energy between the characteristic and the general radiation appears to be nearly constant and independent of the voltage. The experiments of Brainin ${ }^{1}$ show that the total radiation (characteristic plus general) from molybdenum increases as the square of the applied voltage. The experiments of Wooten show that

1 Phys. Rev., Nov., I9I7. 
the characteristic radiation increases approximately according to the same law. It follows that the partition of energy between the two types is constant independent of the voltage.

\section{X-Radiation from Thin Films.}

D. L. Webster ${ }^{1}$ has proposed experiments with thin films for the purpose of studying the nature of the general or "white" X-radiation. An investigation of the emission of characteristic (line) radiation from thin films would also be of interest and value.

The rapid increase of the emitted energy with increase of voltage may be regarded as due to two causes:

(a) The increase in the emission from each atomic nucleus due to the increased energy of the impacting electrons.

(b) The penetration of the electrons into deeper layers of the target with sufficient energy to excite the radiation.

In the case of thin films, if the velocity of the electrons at entrance to the film is sufficiently great they may pass completely through the film and emerge on the far side with sufficient energy still to produce radiation. The part of the radiation that would have been produced by these electrons after passing through the film will be absent and the curve representing the emitted energy will have a break at this point.

A convenient method of investigating this by experiment would be to deposit a thin film of the metal of known thickness upon another metal as a support. If the frequency of the characteristic radiation of the solid supporting target differs from that of the film, the line radiation of the supporting element will not enter the slit of the spectrometer. The radiation from films much thinner than could be independently supported may thus be readily investigated.

From such a film as the voltage is increased the radiated energy will at first increase rapidly in intensity. When the voltage becomes such that the electrons emerge from the far side with an energy greater than $\left(V_{0} e\right)$, the increase in radiation will not be so rapid. That part of the emission due to deeper penetration at this voltage will be absent. There will be a change in the slope of the radiation curve as indicated at $b$, Fig. 3. This will be true independently of any special theory of X-ray emission other than that a portion of the increased emission is due to deeper penetration of the electrons into the target. This phenomenon furnishes a convenient method for measuring the decrease of velocity of electrons on passing through matter. Thus the point $b$ in Fig. 3 corresponds to a voltage across the X-ray tube of about 33.5 kilovolts. The

1 Phys. Rev., March, I9I 7. 
electrons entered the film with a velocity corresponding to 33.5 kilovolts, and emerged from the far side with a velocity corresponding to $V_{0}=19.2$ kilovolts. These results are calculated for a film of molybdenum $5 \times 10^{-4} \mathrm{~cm}$. thick using the value of the constant $b$ previously deduced from Whiddington's experiments.

The form of the radiation curve for a thin film may be readily derived in a similar manner to that for a solid target.

Consider a film of thickness $d$ deposited on a solid support. The radiation will be that from a solid target of the same material as the film less that emitted by the portion of the solid target lying at a depth greater than $d$.

$$
I_{f}=E_{\alpha}\left(h n_{\alpha}\right) B N\left[\int_{0}^{R} \frac{V_{x}-V_{0}}{V_{x}} e^{-\mu c x} d x-e^{-\mu c d} \int_{0}^{R} \frac{V_{x_{1}}-V_{0}}{V_{x_{1}}} e^{\mu c x_{1}} d x_{1}\right] .
$$

The depth of the electron within the surface of the solid portion of the target is designated by $x_{1}$. All of the radiation produced in the $x_{1}$ region passes through the film of constant thickness $d$, hence the absorption due to the film $d$ may be placed outside the integral sign.

The first term of the right-hand member is the same as equation (5). Let $I$ represent this term. Let $I_{s}$ represent the second term.

$$
\begin{gathered}
I_{f}=I-I_{s}, \\
I_{s}=E_{a}\left(h n_{a}\right) B N \int_{0}^{R} \frac{V_{x_{1}}-V_{0}}{V_{x_{1}}} e^{-\mu c x_{1}} d x_{1} .
\end{gathered}
$$

Introducing the law of decrease of electron velocity

$$
V_{x_{1}}{ }^{2}=V_{d}^{2}-b x
$$

and expanding the exponential, the equation is integrated as in the case of $(7)$.

The voltage corresponding to the velocity with which the electrons emerge from under side of film and enters the $X_{1}$ region is designated by $V_{d}$. The range $R$ of the electrons in the $X_{1}$ region is

The $R$ limit is

$$
V_{0}^{2}=V_{d}^{2}-b R
$$

$$
R=\frac{V_{d}^{2}-V_{0}^{2}}{b} .
$$

The integral of (9) for the specified limit is

$$
\begin{aligned}
I_{s}= & E_{a}\left(h n_{a}\right) \frac{B N}{b} e^{-\mu c d}\left[\frac{b}{c \mu}\left(\mathrm{I}-e^{-(c \mu / b)\left(V_{d}{ }^{2}-V_{0}{ }^{2}\right)}\right)\right. \\
& -2 V_{0}\left(V_{d}-V_{0}\right)+2 / 3 \frac{c \mu}{b} V_{0}\left\{2 V_{d}^{3}-V_{0}\left(V_{d}^{2}+V_{0}^{2}\right)\right\}
\end{aligned}
$$




$$
\begin{aligned}
-\frac{V_{0}}{\mathrm{I} 5}\left(\frac{c \mu}{b}\right)^{2}\left\{8 V_{d}^{4}\left(V_{d}-V_{0}\right)-4 V_{d^{2}} V_{0}\left(V_{d}^{2}-V_{0}^{2}\right)\right. & \\
& \left.\left.-3 V_{0}\left(V_{d}^{2}-V_{0}^{2}\right)^{2}\right\}\right] .
\end{aligned}
$$

The value of $V_{d}$ is given by the relation

$$
V_{d}^{2}=V^{2}-b d
$$

where $V$ is the voltage applied to the $\mathrm{X}$-ray tube.

The above equation is zero for $V_{d}=V_{0}$ as it should be. It is to be calculated only for values of $V_{d}$ greater than $V_{0}$. A plot of this equation is shown at $n m p$ in Fig. 3. The constants $b$ and $\mu$ are given the same

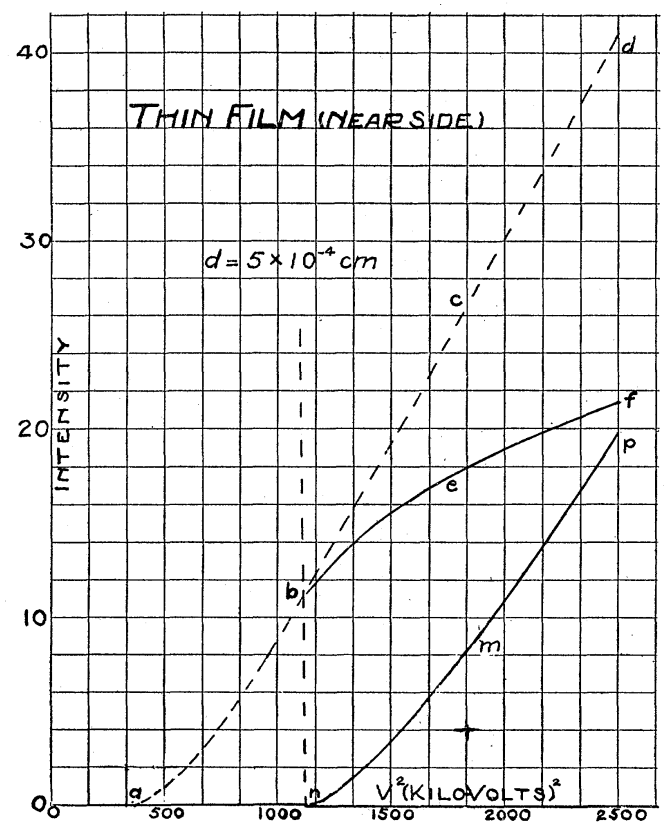

Fig. 3.

values as in the plot of Fig. 2. The thickness of the film is taken to be $d=5 \dot{\times} \mathrm{IO}^{-4} \mathrm{~cm}$.

This curve is subtracted from the curve $a b c d$ as indicated by (8). The resultant curve $a b$ ef represents the radiation from a thin film.

The equation and its plot indicate that the radiation could not indefinitely increase, but at a high voltage it would approach a maximum independent of the voltage. Consideration of the physical processes involved would also lead one to anticipate this result.

An inspection of equation (7) for large values of $\mu$ (that is very soft 
rays) shows that the radiation from a solid target (Fig. 2) would not indefinitely increase with the voltage. The energy emitted will tend toward a maximum independent of the voltage. This result is also to be expected from a consideration of the physical processes involved. In the case of elements such as copper, chromium, etc., which have a characteristic radiation of long wave-lengths, the radiation emitted from atoms at considerable depths below the surface would be largely absorbed in the target. The resultant emission would be similar to that from thin films. The above remarks are true in a degree for the characteristic emission from all elements. The energy radiated cannot increase indefinitely with the voltage. This is necessarily true, otherwise at extremely high voltages the radiated energy might become greater than that of the bombarding electron stream-a result that is manifestly impossible.

\section{Emission from Thin Films. (FAr Side.)}

Another matter of interest is the emission of the characteristic radiation from the far side of a thin film. The expression for the radiation for this case is readily derived by the methods pursued in the previous developments.

Let the thickness of the film be designated by $d$. The thickness of material traversed by the $\mathrm{X}$-rays emitted from any depth $x$ and emerging from the far side will be $(d-x)$.

The radiation may be represented by

Since

$$
I=E_{a}\left(h n_{a}\right) B N \int_{0}^{R} \frac{V_{x}-V_{0}}{V_{x}} e^{-\mu(d-x)} d x .
$$

$$
\begin{gathered}
V_{x}^{2}=V^{2}-b x \\
I=E_{a}\left(h n_{\alpha}\right) B N e^{-\mu d}\left[\int_{0}^{R} e^{\mu x} d x-V_{0} \int_{0}^{R} \frac{e^{\mu x} d x}{\left(V^{2}-b x\right)^{1 / 2}}\right] .
\end{gathered}
$$

The constants and the limit $R$ have the same significance as in the equation for a solid target.

The integral equation is

$$
\begin{aligned}
I & =E_{a}\left(h n_{a}\right) B N \frac{e^{-\mu d}}{b}\left[\frac{b}{\mu}\left(e^{-(\mu / b)\left(V^{2}-V_{0}^{2}\right)}-\mathrm{I}\right)\right. \\
& -2 V_{0}\left(V-V_{0}\right)-\frac{2}{3} \frac{\mu}{b} V_{0}\left\{2 V^{3}-V_{0}\left(V^{2}+V_{0}^{2}\right)\right\} \\
& \left.-\frac{\mathrm{I}}{\mathrm{I}}\left(\frac{\mu}{b}\right)^{2} V_{0}\left\{8 V^{4}\left(V-V_{0}\right)-4 V^{2} V_{0}\left(V^{2}-V_{0}^{2}\right)-3 V_{0}\left(V^{2}-V_{0}^{2}\right)^{2}\right\}\right] .
\end{aligned}
$$


A plot of this equation is shown in Fig. 4. It is to be noticed that the curve is much more concave upward than the curve for the emission from a solid target (Fig. 2). The variable part of the equation does not con-

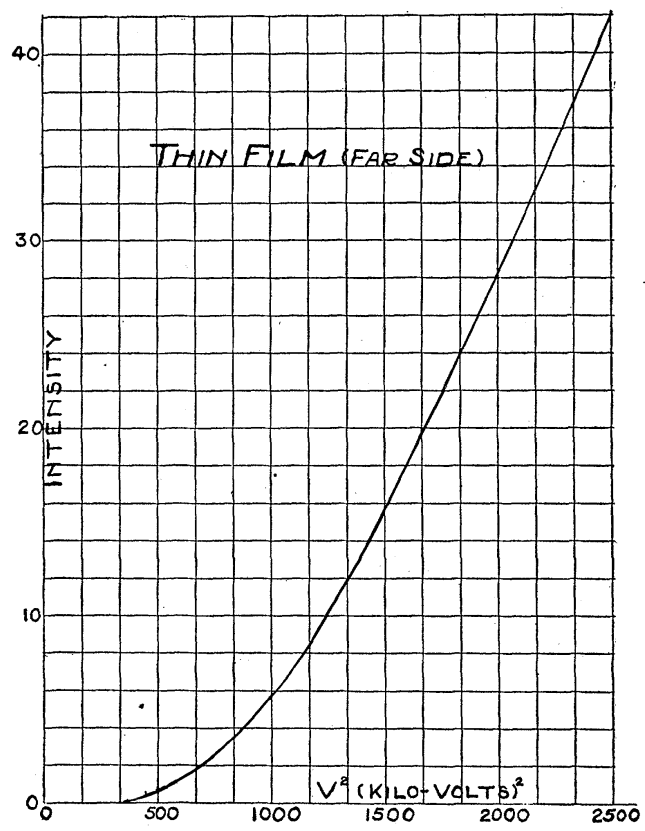

Fig. 4.

tain the term involving the thickness of the film. The form of the radiation curve is independent of the thickness of the film provided this thickness is greater than the range $R$ of the electrons producing the radiations.

Phonix Physical Laboratory,

Columbia University,

January, 1918. 